\title{
Sobre o Decaimento Exponencial da Energia de um sistema Termoelástico
}

\author{
On the Exponential Decay of the Energy of a Thermoelastic System
}

\author{
Débora Dalmolin ${ }^{1}$ e Marcio Violante Ferreira ${ }^{2}$ \\ ${ }^{1}$ Aluna do PPGMat/UFSM, Universidade Federal de Santa Maria, Santa Maria, RS, Brasil \\ deborasdalmolin@hotmail.com \\ ${ }^{2}$ Professor do Departamento de Matemática, Universidade Federal de Santa Maria, Santa Maria, RS, Brasil \\ marcio.ferreira@ufsm.br
}

\begin{abstract}
Resumo
Neste trabalho estudamos a existência, unicidade e o comportamento assintótico da solução de um sistema termoelástico linear em dimensão um. Tal sistema é composto por uma equação da onda acoplada com a equação do calor e, fisicamente, modela a ação recíproca entre as vibrações de uma corda elástica e a variação de sua temperatura. Provamos, inicialmente, a existência e unicidade de solução do sistema fazendo uso da Teoria de Semigrupos de Operadores Lineares Limitados. Para a análise do comportamento assintótico da solução, utilizamos um método que consiste em perturbar adequadamente a energia do sistema e, com isso, provamos que a energia total do sistema decai exponencialmente a zero quando $t \rightarrow \infty$.
\end{abstract}

Palavras-chave: Sistema termoelástico, teoria de semigrupos, decaimento exponencial.

\begin{abstract}
In this paper we study the existence, uniqueness and the asymptotic behavior of the solution of a one dimensional linear thermoelastic system. Such a system is composed of a wave equation coupled with the heat equation and, physically, model the interaction of the vibrations of an elastic string with the variation of its temperature. We prove, first, the existence and uniqueness of solution of the system making use of the Theory of Semigroups of Bounded Linear Operators. For the analysis of the asymptotic behavior of the solution, we use a method consisting of a suitably perturbation to the energy of the system and, with that, we prove that the total energy of the system decays exponentially to zero as $t \rightarrow \infty$.
\end{abstract}

Keywords: Thermoelastic system, semigroups theory, exponential decay. 


\section{Introdução}

Consideramos neste trabalho o sistema acoplado de equações diferenciais parciais

$$
\begin{array}{r}
u_{t t}-u_{x x}+\sigma(x) u_{t}+\alpha \theta_{x}=0, \\
\theta_{t}-\theta_{x x}+\beta u_{x t}=0,
\end{array}
$$

para $0<x<L$ e $0<t<+\infty$, complementado pelas condições iniciais

$$
\begin{array}{r}
u(x, 0)=u_{0}(x), u_{t}(x, 0)=u_{1}(x), \\
\theta(x, 0)=\theta_{0}(x), \text { em }(0, L),
\end{array}
$$

e condições de fronteira

$$
u(0, t)=u(L, t)=\theta(0, t)=\theta(L, t)=0, \quad t>0,
$$

onde $\alpha$ e $\beta$ são constantes de acoplamento reais estritamente positivas. Além disso, supõe-se que $\sigma(x) \in$ $L^{\infty}(0, L), \mathrm{com}$

$$
0<\sigma_{0} \leq \sigma(x) \leq \sigma_{1} \text {, q.s. em }(0, L),
$$

onde $\sigma_{0}$ e $\sigma_{1}$ são constantes.

Como pode ser visto em Nowacki (1962), Achenbach (1973) e Ciarlet (1993), o sistema de equações acima modela a ação recíproca entre as vibrações (deformações) de uma corda elástica de comprimento $L$ (cuja amplitude do movimento é dada pela função $u(x, t))$ e a variação de temperatura em cada ponto da mesma em relação a uma temperatura referencial fixa (variação esta dada pela função $\theta(x, t))$. A função $\sigma(x)$, que aparece em (1), representa um mecanismo de dissipação ("damping") interna variável.

A Energia Total do sistema (1)-(4) é dada por

$$
\mathcal{E}(t)=\frac{1}{2} \int_{0}^{L}\left(u_{t}^{2}+u_{x}^{2}+\frac{\alpha}{\beta} \theta^{2}\right) d x
$$

e um cálculo direto nos dá, pelo menos formalmente,

$$
\frac{d}{d t} \mathcal{E}(t)=-\int_{0}^{L} \sigma(x) u_{t}^{2} d x-\frac{\alpha}{\beta} \int_{0}^{L} \theta_{x}^{2} d x \leq 0,
$$

o que mostra que a Energia decresce com o passar do tempo. O objetivo principal do nosso trabalho é, justamente, mostrar que $\mathcal{E}(t) \rightarrow 0$ exponencialmente quando $t \rightarrow+\infty$.

Dado o grande interesse em se enterder matematicamente fenômenos físicos como o descrito pelo sistema (1)-(4), é vasta a literatura que trata do assunto. Podemos citar, por exemplo, o trabalho de Dafermos (1968), que deu substanciais contribuições para o estudo da existência de soluções bem como da estabilidade assintótica das soluções do sistema de equações em termoelasticidade linear, em dimensão três. Para este mesmo problema, mas supondo que a força restauradora é proporcional ao vetor velocidade, Pereira e Menzala (1989) provaram que, num meio isotrópico e não-homogêneo, a energia total associada ao sistema decai exponencialmente a zero quando $t \rightarrow+\infty$. Ressalte-se que, neste trabalho, os autores consideraram $\sigma$ como constante.

No caso em que se considera o sistema termoelástico linear em dimensão um, Rivera (1992) obteve resultados de decaimento exponencial para a energia, até terceira ordem, do problema (1)-(4), mas sem considerar mecanismos de dissipação interna, isto é, analisou o caso em que $\sigma \equiv 0$. O resultado obtido é de extrema relevância, haja vista que só a dissipação térmica é suficiente para a estabilização assintótica da energia. No entando, o autor considerou dados iniciais com bem mais regularidade do que a considerada no presente trabalho. Além disso, a prova da existência e unicidade de solução de (1)-(4) que apresentamos aqui difere em alguns aspectos (ferramentas de análise funcional utilizadas) da apresentada por Rivera (1992).

A análise matemática do comportamento da energia total é, obviamente, de grande interesse para outros sistemas de equações diferenciais parciais, que modelam diferentes fenômenos físicos. Por exemplo, utilizando multiplicadores adequados, Ferreira e Menzala (2006) obtiveram a estabilização da solução de um sistema de ondas elásticas semi-linear em domínios exteriores e, em Ferreira e Menzala (2007), resultados análogos para um sistema elasto-eletromagnético não-linear. Obviamente há, nesses casos em que se considera fenômenos em domínios exteriores, a dificuldade adicional de se trabalhar numa região não limitada do espaço.

Problemas ainda mais gerais podem ser estudados, como aqueles em que se consideram mecanismos de dissipação na fronteira ou mesmo dissipação localizada, isto é, atuando apenas numa parte do domínio. Os resultados que apresentamos neste trabalho são um caminho para tratar estes casos e outros ainda mais gerais como, por exemplo, o problema não-linear associado ao modelo (1)-(4).

Nosso trabalho está estruturado da seguinte forma: utilizando a teoria de semigrupos de operadores lineares provamos, na próxima seção, a existência e unicidade de solução de (1)-(4). Na Seção 3, utilizando um funcional adequado para perturbar a energia do sistema, provamos que a mesma decai exponencialmente no tempo. As conclusões e as referências encerram o texto.

\section{Existência e unicidade de solução}

Inicialmente, faremos uma breve apresentação dos espaços funcionais utilizados ao longo deste trabalho. Para maiores esclarecimentos sobre a notação e propri- 
edades utilizadas em algumas demonstrações, pode-se consultar Adams (1975) e Brezis (2010).

O produto interno e norma usuais no espaço $L^{2}(0, L)$ serão denotados, respectivamente, por (. . .) e $|$.$| . Assim,$

$$
(u, v)=\int_{0}^{L} u v d x, \quad \forall u, v \in L^{2}(0, L)
$$

e

$$
|u|=\left(\int_{0}^{L} u^{2} d x\right)^{\frac{1}{2}}, \forall u \in L^{2}(0, L) .
$$

Como se sabe, $L^{2}(0, L)$, munido do produto interno acima, é um espaço de Hilbert.

Usaremos a notação tradicional $\mathcal{D}(0, L)$ para o espaço das funções testes, definidas em $(0, L)$, e $\mathcal{D}^{\prime}(0, L)$ para o espaço das distribuições. Os espaços de Sobolev clássicos serão representados por $H^{m}(0, L)$, $m$ um número inteiro positivo. No subespaço $H_{0}^{1}(0, L)$ (fecho de $\mathcal{D}(0, L)$ em $\left.H^{1}(0, L)\right)$ vale a Desigualdade de Poincaré

$$
\int_{0}^{L} u^{2} d x \leq C_{0} \int_{0}^{L} u_{x}^{2} d x
$$

Portanto, em $H_{0}^{1}(0, L)$, considera-se o produto interno e norma equivalentes a de $H^{1}(0, L)$ dados por

$$
((u, v))=\int_{0}^{L} u_{x} v_{x} d x, \quad \forall u, v \in H_{0}^{1}(0, L)
$$

e

$$
\|u\|=\left(\int_{0}^{L} u_{x}^{2} d x\right)^{\frac{1}{2}}, \forall u \in H_{0}^{1}(0, L) .
$$

A existência e unicidade de solução do sistema (1)-(4) será estabelecida via Teoria de Semigrupos de Operadores Lineares. A ideia, então, é reescrevê-lo na forma de um problema abstrato de primeira ordem. Para isso, introduzimos o espaço

$$
\mathcal{H}=H_{0}^{1}(0, L) \times L^{2}(0, L) \times L^{2}(0, L)
$$

que, obviamente, é um espaço de Hilbert com o produto interno

$$
\begin{aligned}
\left(\left(u_{1}, v_{1}, \theta_{1}\right),\left(u_{2}, v_{2}, \theta_{2}\right)\right)_{\mathcal{H}} & =\int_{0}^{L}\left(u_{1}\right)_{x}\left(u_{2}\right)_{x} d x \\
& +\int_{0}^{L} v_{1} v_{2} d x+\int_{0}^{L} \theta_{1} \theta_{2} d x
\end{aligned}
$$

$\operatorname{com}\left(u_{1}, v_{1}, \theta_{1}\right),\left(u_{2}, v_{2}, \theta_{2}\right) \in \mathcal{H}$.
O sistema (1)-(2), com condições iniciais (3), pode ser reescrito (via a substituição $v=u_{t}$ na equação (1)) na forma

$$
\left\{\begin{array}{l}
\frac{d U}{d t}=\mathcal{A U} \\
U(0)=U_{0}
\end{array}\right.
$$

onde $U(t)=(u(t), v(t), \theta(t)), U_{0}=\left(u_{0}, u_{1}, \theta_{0}\right)$ e $\mathcal{A}$ : $D(\mathcal{A}) \subset \mathcal{H} \rightarrow \mathcal{H}$ é o operador linear não-limitado com domínio

$$
\begin{aligned}
D(\mathcal{A}) & =H^{2}(0, L) \cap H_{0}^{1}(0, L) \times H_{0}^{1}(0, L) \\
& \times H^{2}(0, L) \cap H_{0}^{1}(0, L)
\end{aligned}
$$

definido por

$$
\mathcal{A}(u, v, \theta)=\left(v, u_{x x}-\sigma(x) v-\alpha \theta_{x},-\beta v_{x}+\theta_{x x}\right),
$$

para todo $(u, v, \theta) \in D(\mathcal{A})$.

Observemos que não se pode garantir que o operador $\mathcal{A}$ é dissipativo. Com efeito, se $U=(u, v, \theta) \in D(\mathcal{A})$, então

$$
\begin{aligned}
(\mathcal{A} U, U)_{\mathcal{H}} & =\int_{0}^{L} v_{x} u_{x} d x+\int_{0}^{L} u_{x x} v d x-\int_{0}^{L} \sigma(x) v^{2} d x \\
& -\alpha \int_{0}^{L} v \theta_{x} d x-\beta \int_{0}^{L} v_{x} \theta d x+\int_{0}^{L} \theta_{x x} \theta d x .
\end{aligned}
$$

Daí, notando que,

$$
\begin{gathered}
\int_{0}^{L} v_{x} u_{x} d x+\int_{0}^{L} u_{x x} v d x=\int_{0}^{L} \frac{\partial}{\partial x}\left(u_{x} v\right) d x=0, \\
\int_{0}^{L} v_{x} \theta d x=-\int_{0}^{L} v \theta_{x} d x
\end{gathered}
$$

e

$$
\int_{0}^{L} \theta_{x x} \theta d x=-\int_{0}^{L} \theta_{x}^{2} d x
$$

já que $v, \theta \in H_{0}^{1}(0, L)$, obtemos

$$
\begin{aligned}
(\mathcal{A} U, U)_{\mathcal{H}}= & -\int_{0}^{L} \sigma(x) v^{2} d x+(\beta-\alpha) \int_{0}^{L} v \theta_{x} d x \\
& -\int_{0}^{L} \theta_{x}^{2} d x .
\end{aligned}
$$

Ainda, utilizando a Desigualdade de Young e a hipótese (5), segue que

$$
(\mathcal{A} U, U)_{\mathcal{H}} \leq-\sigma_{0} \int_{0}^{L} v^{2} d x+\frac{1}{2}(\beta-\alpha)^{2} \int_{0}^{L} v^{2} d x
$$




$$
-\frac{1}{2} \int_{0}^{L} \theta_{x}^{2} d x
$$

e, portanto,

$$
(\mathcal{A} U, U)_{\mathcal{H}} \leq-\sigma_{0}|v|^{2}+\frac{1}{2}(\beta-\alpha)^{2}|| U\left\|_{\mathcal{H}}^{2}-\frac{1}{2}\right\| \theta \|^{2} .
$$

A desigualdade anterior nos leva a considerar uma perturbação limitada do operador $\mathcal{A}$, escolhida adequadamente, de modo que o novo operador seja dissipativo (conforme Pazy (1983), pg. 79). Consideremos, pois, o operador $\mathcal{B}: D(\mathcal{B}) \subset \mathcal{H} \rightarrow \mathcal{H}$, definido por

$$
\mathcal{B}=-(\alpha-\beta)^{2} I+\mathcal{A},
$$

onde $D(\mathcal{B})=D(\mathcal{A})$ e $I$ é o operador identidade em $\mathcal{H}$.

Proposição 2.1. $D(\mathcal{B})$ é denso em $\mathcal{H}$.

Demonstração Segue diretamente da cadeia de inclusões

$$
\begin{aligned}
& \mathcal{D}(0, L) \subset H^{2}(0, L) \cap H_{0}^{1}(0, L) \subset H_{0}^{1}(0, L), \\
& \mathcal{D}(0, L) \subset H_{0}^{1}(0, L) \subset L^{2}(0, L), \\
& \mathcal{D}(0, L) \subset H^{2}(0, L) \cap H_{0}^{1}(0, L) \subset L^{2}(0, L),
\end{aligned}
$$

e das densidades de $\mathcal{D}(0, L)$ em $H_{0}^{1}(0, L)$ e de $\mathcal{D}(0, L)$ em $L^{2}(0, L)$.

Proposição 2.2. O operador $\mathcal{B}$ é dissipativo, isto é,

$$
(\mathcal{B} U, U)_{\mathcal{H}} \leq 0, \forall U=(u, v, \theta) \in D(\mathcal{B}) .
$$

Demonstração Se $U=(u, v, \theta) \in D(\mathcal{B})$, então

$$
\begin{aligned}
(\mathcal{B} U, U)_{\mathcal{H}} & =\left(\left(-(\alpha-\beta)^{2} I+\mathcal{A}\right) U, U\right)_{\mathcal{H}} \\
& =-(\alpha-\beta)^{2}\|U\|_{\mathcal{H}}^{2}+(\mathcal{A} U, U)_{\mathcal{H}} .
\end{aligned}
$$

Disto, e da desigualdade (7), obtem-se

$$
\begin{aligned}
(\mathcal{B} U, U)_{\mathcal{H}} & \leq-(\alpha-\beta)^{2}|| U \|_{\mathcal{H}}^{2}-\sigma_{0}|v|^{2} \\
& +\frac{1}{2}(\beta-\alpha)^{2}|| U\left\|_{\mathcal{H}}^{2}-\frac{1}{2}|| \theta\right\|^{2},
\end{aligned}
$$

ou seja,

$$
(\mathcal{B} U, U)_{\mathcal{H}} \leq-\left.\frac{1}{2}(\alpha-\beta)^{2}|| U\right|_{\mathcal{H}} ^{2}-\sigma_{0}|v|^{2}-\frac{1}{2}|| \theta \|^{2} \leq 0,
$$

o que prova o desejado.

Proposição 2.3. I-B é um operador sobrejetivo, isto é, $\mathcal{R}(I-\mathcal{B})=\mathcal{H}$, onde I é o operador identidade em $\mathcal{H}$.

Demonstração Devemos mostrar que para todo $G=$ $(f, g, h) \in \mathcal{H}$, existe $U=(u, v, \theta) \in D(\mathcal{B})$ tal que

$$
(I-\mathcal{B}) U=G,
$$

ou seja, devemos resolver o sistema

$$
\left\{\begin{array}{l}
{\left[(\alpha-\beta)^{2}+1\right] u-v=f} \\
{\left[(\alpha-\beta)^{2}+1\right] v-u_{x x}+\sigma(x) v+\alpha \theta_{x}=g} \\
{\left[(\alpha-\beta)^{2}+1\right] \theta+\beta v_{x}-\theta_{x x}=h,}
\end{array}\right.
$$

onde $f \in H_{0}^{1}(0, L), g \in L^{2}(0, L)$ e $h \in L^{2}(0, L)$ são funções dadas.

Chamando $k=\left[(\alpha-\beta)^{2}+1\right]$, sendo $v=k u-f$ e $v_{x}=k u_{x}-f_{x}$, o sistema (9) se resume a encontrar $u, \theta \in H^{2}(0, L) \cap H_{0}^{1}(0, L)$ tais que

$$
\left\{\begin{array}{l}
\left(k^{2}+\sigma(x) k\right) u-u_{x x}+\alpha \theta_{x}=(k+\sigma(x)) f+g \\
k \theta+\beta k u_{x}-\theta_{x x}=\beta f_{x}+h,
\end{array}\right.
$$

com $f \in H_{0}^{1}(0, L), g \in L^{2}(0, L)$ e $h \in L^{2}(0, L)$ funções dadas. Assim, resolvendo (10), obtemos a solução procurada $(u, v, \theta) \in D(\mathcal{B})$ do problema (9) e, consequentemente, resolvemos (8).

Com o intuito de resolver o sitema (10), vamos considerar o espaço de Hilbert

$$
\mathcal{W}=H_{0}^{1}(0, L) \times H_{0}^{1}(0, L),
$$

a forma bilinear

$$
a: \mathcal{W} \times \mathcal{W} \rightarrow \mathbb{R},
$$

definida por

$$
\begin{aligned}
& a((u, \theta),(\psi, \phi))=k \beta \int_{0}^{L}\left(k^{2}+\sigma(x) k\right) u \psi d x \\
& +k \beta \int_{0}^{L} u_{x} \psi_{x} d x+k \beta \alpha \int_{0}^{L} \theta_{x} \psi d x+\alpha k \int_{0}^{L} \theta \phi d x \\
& +\alpha \beta k \int_{0}^{L} u_{x} \phi d x+\alpha \int_{0}^{L} \theta_{x} \phi_{x} d x
\end{aligned}
$$

e a forma linear

$$
F: \mathcal{W} \rightarrow \mathbb{R}
$$

dada por

$$
\begin{aligned}
F(\psi, \phi) & =k \beta \int_{0}^{L}(k+\sigma(x)) f \psi d x+k \beta \int_{0}^{L} g \psi d x \\
& +\alpha \beta \int_{0}^{L} f_{x} \phi d x+\alpha \int_{0}^{L} h \phi d x .
\end{aligned}
$$

Notemos que, para $(u, \theta) \in \mathcal{W}$ qualquer, temos

$$
a((u, \theta),(u, \theta))=k \beta \int_{0}^{L}\left(k^{2}+\sigma(x) k\right) u^{2} d x
$$




$$
\begin{array}{r}
+k \beta \int_{0}^{L} u_{x}^{2} d x+k \beta \alpha \int_{0}^{L} \theta_{x} u d x \\
+\alpha k \int_{0}^{L} \theta^{2} d x+\alpha \beta k \int_{0}^{L} u_{x} \theta d x+\alpha \int_{0}^{L} \theta_{x}^{2} d x,
\end{array}
$$

donde, integrando por partes e usando a hipótese (5), obtemos

$$
\begin{aligned}
& a((u, \theta),(u, \theta)) \geq k \beta\left(k^{2}+\sigma_{0} k\right) \int_{0}^{L} u^{2} d x \\
& +k \beta \int_{0}^{L} u_{x}^{2} d x+\alpha k \int_{0}^{L} \theta^{2} d x+\alpha \int_{0}^{L} \theta_{x}^{2} d x \\
& \geq k \beta \int_{0}^{L} u_{x}^{2} d x+\alpha \int_{0}^{L} \theta_{x}^{2} d x
\end{aligned}
$$

ou seja,

$$
\begin{aligned}
a((u, \theta),(u, \theta)) & \geq C\left(\|u\|^{2}+\|\theta\|^{2}\right) \\
& =C\|(u, \theta)\|_{\mathcal{W}}^{2}
\end{aligned}
$$

onde $C=\min \{k \beta, \alpha\}$, o que mostra que $a$ é coerciva. Além disso, a é contínua. Com efeito, graças à hipótese (5) e a Desigualdade de Cauchy-Schwarz obtemos, para quaisquer $(u, \theta),(\psi, \phi) \in \mathcal{W}$,

$$
\begin{aligned}
& |a((u, \theta),(\psi, \phi))| \leq k \beta\left(k^{2}+\sigma_{1} k\right)|u\|\psi \mid+k \beta\| u\|\| \psi \| \\
& +k \beta \alpha\|\theta\||\psi|+\alpha k|\theta||\phi|+\alpha \beta k\|u\||\phi|+\alpha\|\theta\|\|\phi\|
\end{aligned}
$$

e, pela Desigualdade de Poincaré,

$$
\begin{aligned}
& |a((u, \theta),(\psi, \phi))| \leq \\
& \leq C_{1}(\|u\|\|\psi\|+\|\theta\|\|\psi\|+\|u\|\|\phi\|+\|\theta\|\|\phi\|) \\
& \leq C_{2}\left(\|u\|^{2}+\|\theta\|^{2}\right)^{\frac{1}{2}}\left(\|\psi\|^{2}+\|\phi\|^{2}\right)^{\frac{1}{2}} \\
& =C_{2}\|(u, \theta)\|_{\mathcal{W}}\|(\psi, \phi)\|_{\mathcal{W} .}
\end{aligned}
$$

Finalmente, observemos que, para todo $(\psi, \phi) \in \mathcal{W}$, vale, graças à Desigualdade de Cauchy-Schwarz,

$$
\begin{aligned}
|F(\psi, \phi)| & \leq k \beta\left(k+\sigma_{1}\right)|f||\psi|+k \beta|g||\psi| \\
& +\alpha \beta\|f\||\phi|+\alpha|h||\phi| \\
& =k \beta\left[\left(k+\sigma_{1}\right)|f|+|g|\right]|\psi|+\alpha[\beta\|f\|+|h|]|\phi| \\
& =C_{3}|\psi|+C_{4}|\phi| \leq C_{5}\left(|\psi|^{2}+|\phi|^{2}\right)^{\frac{1}{2}}
\end{aligned}
$$

de onde se obtém, utilizando a Desigualdade de Poincaré,

$$
|F(\psi, \phi)| \leq C_{6}\left(\|\psi\|^{2}+\|\phi\|^{2}\right)^{\frac{1}{2}}=C_{6}\|(\psi, \phi)\|_{\mathcal{W}}
$$

isto válido para todo $(\psi, \phi) \in \mathcal{W}$, o que significa que $F$ é contínua.
Mostramos, até aqui, que a forma bilinear $a$ é contínua e coerciva e a forma linear $F$ é contínua. Então, pelo Teorema de Lax-Milgram (veja Brezis (2010)), existe único $(u, \theta) \in \mathcal{W}=H_{0}^{1}(0, L) \times H_{0}^{1}(0, L)$ tal que

$$
a((u, \theta),(\psi, \phi))=F(\psi, \phi),
$$

para todo $(\psi, \phi) \in \mathcal{W}$. Em particular, vale a identidade (11) para todo $(\psi, 0)$, com $\psi \in \mathcal{D}(0, L)$. Assim, no sentido das distribuições,

$$
\begin{array}{r}
\left\langle\left(k^{2}+\sigma(x) k\right) u, \psi\right\rangle+\left\langle u_{x}, \psi_{x}\right\rangle+\alpha\left\langle\theta_{x}, \psi\right\rangle \\
=\langle(k+\sigma(x)) f, \psi\rangle+\langle g, \psi\rangle,
\end{array}
$$

$\forall \psi \in \mathcal{D}(0, L)$ e, portanto, vale em $\mathcal{D}^{\prime}(0, L)$ a igualdade

$$
u_{x x}=\left(k^{2}+\sigma(x) k\right) u+\alpha \theta_{x}-(k+\sigma(x)) f-g .
$$

Mas $u \in H_{0}^{1}(0, L), \theta \in H_{0}^{1}(0, L)$, as funções dadas $f \in H_{0}^{1}(0, L), g \in L^{2}(0, L)$ e $\sigma(x) u \in L^{2}(0, L)$, donde conclui-se que $u_{x x} \in L^{2}(0, L)$ e, consequentemente, $u \in H^{2}(0, L) \cap H_{0}^{1}(0, L)$.

Definamos agora

$$
v=k u-f .
$$

Então $v \in H_{0}^{1}(0, L) \mathrm{e}$

$$
k u-v=f .
$$

Além disso, como

$$
k^{2} u+\sigma(x) k u-u_{x x}+\alpha \theta_{x}=k f+\sigma(x) f+g,
$$

teremos

$$
k v+\sigma(x) v-u_{x x}+\alpha \theta_{x}=g .
$$

Por outro lado, vale a identidade (11) para todo $(0, \phi)$, $\operatorname{com} \phi \in \mathcal{D}(0, L)$. Assim, no sentido das distribuições,

$$
k\langle\theta, \phi\rangle+\beta k\left\langle u_{x}, \phi\right\rangle+\left\langle\theta_{x}, \phi_{x}\right\rangle=\beta\left\langle f_{x}, \phi\right\rangle+\langle h, \phi\rangle,
$$

para toda $\phi \in \mathcal{D}(0, L)$ e, assim,

$$
\theta_{x x}=k \theta+\beta k u_{x}-\beta f_{x}-h,
$$

igualdade essa no sentido de $\mathcal{D}^{\prime}(0, L)$. Como $u, \theta, f \in$ $H_{0}^{1}(0, L)$ e $h \in L^{2}(0, L)$, conclui-se que $\theta_{x x} \in L^{2}(0, L)$. Portanto $\theta \in H^{2}(0, L) \cap H_{0}^{1}(0, L)$ e

$$
k \theta+\beta v_{x}-\theta_{x x}=h .
$$

Em resumo, mostramos a existência de $(u, v, \theta) \in$ $D(\mathcal{B})$ satisfazendo as equações (9), o que encerra a demonstração da proposição.

Provamos, até aqui, que $\mathcal{B}$ é um operador densamente definido e maximal dissipativo. Segue então, do Teorema de Lumer-Phillips (veja Pazy (1983)), que $\mathcal{B}$ é o gerador infinitesimal de um semigrupo de contrações de Classe $C_{0}$. 
Ora, como o operador $(\alpha-\beta)^{2} I$ é linear e limitado, podemos utilizar a Teoria da Perturbação de Semigrupos (Pazy (1983), pg. 79) para garantir que o operador $\mathcal{B}+$ $(\alpha-\beta)^{2}=\mathcal{A}$ é o gerador infinitesimal de um semigrupo de Classe $C_{0}$. Assim, $\mathcal{A}$ é o gerador infinitesimal de um semigrupo $\{S(t)\}_{t \geq 0}$ de Classe $C_{0}$ e $U(t)=S(t) U_{0}$ é a única solução do problema

$$
\left\{\begin{array}{l}
\frac{d}{d t} U(t)=\mathcal{A} U(t) \\
U(0)=U_{0} .
\end{array}\right.
$$

Resta provado, pois, o seguinte resultado de existência de solução para o problema (6) e, consequentemente, para o sistema (1)-(4):

Teorema 2.4. Suponhamos que $\sigma$ satisfaz a hipótese (5). Então, para cada $\left(u_{0}, u_{1}, \theta_{0}\right) \in D(\mathcal{A})$, o sistema (1)-(4) tem uma única solução global forte $(u, \theta)$ tal que

$$
\begin{aligned}
u \in & C\left([0, \infty) ; H^{2}(0, L) \cap H_{0}^{1}(0, L)\right) \cap \\
& \cap C^{1}\left([0, \infty) ; H_{0}^{1}(0, L)\right) \cap C^{2}\left((0, \infty) ; L^{2}(0, L)\right)
\end{aligned}
$$

$e$

$$
\begin{gathered}
\theta \in C\left([0, \infty) ; H^{2}(0, L) \cap H_{0}^{1}(0, L)\right) \cap \\
\cap C^{1}\left((0, \infty) ; L^{2}(0, L)\right) .
\end{gathered}
$$

\section{Comportamento assintótico}

Como uma apresentação do método a ser utilizado para o estudo do comportamento assintótico da solução do sistema (1)-(4), obtida na seção anterior, vamos analisar o comportamento da solução do problema de valor inicial e de contorno

$$
\begin{array}{r}
u_{t t}-u_{x x}+\sigma(x) u_{t}=0,0<x<L \text { e } 0<t<+\infty, \\
u(x, 0)=u_{0}(x), u_{t}(x, 0)=u_{1}(x), 0<x<L, \\
u(0, t)=u(L, t)=0, t>0,
\end{array}
$$

onde a função $\sigma(x) \in L^{\infty}(0, L)$ satisfaz a hipótese (5), isto é,

$$
0<\sigma_{0} \leq \sigma(x) \leq \sigma_{1} \text {, q.s. em }(0, L) .
$$

A existência e unicidade de solução para o problema acima pode ser obtida com aquele mesmo método empregado na seção anterior. De fato, se $\left(u_{0}, u_{1}\right) \in$ $H^{2}(0, L) \cap H_{0}^{1}(0, L) \times H_{0}^{1}(0, L)$, então existe uma única solução global forte $u$ de (12)-(14) tal que

$$
\begin{aligned}
u \in C & \left([0, \infty), H^{2}(0, L) \cap H_{0}^{1}(0, L)\right) \cap \\
& \cap C^{1}\left([0, \infty), H_{0}^{1}(0, L)\right) \cap C^{2}\left((0, \infty), L^{2}(0, L)\right) .
\end{aligned}
$$

A Energia Total associada ao modelo (12)-(14) é dada pela integral

$$
\mathcal{E}(t) \equiv \frac{1}{2} \int_{0}^{L}\left(u_{t}^{2}(x, t)+u_{x}^{2}(x, t)\right) d x
$$

e um cálculo simples nos dá

$$
\begin{aligned}
\frac{d}{d t} \mathcal{E}(t) & =-\int_{0}^{L} \sigma(x) u_{t}^{2}(x, t) d x \\
& \leq-\sigma_{0} \int_{0}^{L} u_{t}^{2}(x, t) d x \leq 0,
\end{aligned}
$$

o que significa que a energia é decrescente e o modelo tem, pois, caráter dissipativo. O próximo resultado mostra que a energia decai exponencialmente a zero quando $t \rightarrow \infty$. A demonstração deste fato se baseia num método que consiste em perturbar adequadamente o funcional energia $\mathcal{E}(t)$.

Teorema 3.1. Seja $\mathcal{E}(t)$ a energia total do sistema (12)-(14), definida pela identidade (15). Então, existem constantes $C, \gamma>0$ tais que

$$
\mathcal{E}(t) \leq C \mathcal{E}(0) \exp (-\gamma t), \forall t \geq 0 .
$$

Demonstração Multiplicando ambos os membros da equação (12) por $u$ e integrando em $(0, L)$ obtemos

$$
\begin{array}{r}
\frac{d}{d t}\left\{\int_{0}^{L} u_{t} u d x+\frac{1}{2} \int_{0}^{L} \sigma(x) u^{2} d x\right\}= \\
-\int_{0}^{L} u_{x}^{2} d x+\int_{0}^{L} u_{t}^{2} d x .
\end{array}
$$

Ponhamos

$$
G(t)=\int_{0}^{L} u_{t} u d x+\frac{1}{2} \int_{0}^{L} \sigma(x) u^{2} d x .
$$

Então, obviamente,

$$
\frac{d}{d t} G(t)=-\int_{0}^{L} u_{x}^{2} d x+\int_{0}^{L} u_{t}^{2} d x .
$$

Agora, definimos o funcional Energia Perturbada

$$
H(t)=\mathcal{E}(t)+\delta G(t)
$$

onde $\delta$ é um parâmetro positivo a ser fixado posteriormente. Levando em conta (16) e (18), vemos que

$$
\frac{d}{d t} H(t) \leq-\delta \int_{0}^{L} u_{x}^{2} d x-\left(\sigma_{0}-\delta\right) \int_{0}^{L} u_{t}^{2} d x .
$$

Se fixarmos $\delta$ suficientemente pequeno de modo que $0<\delta<\sigma_{0}$, obteremos

$$
\frac{d}{d t} H(t) \leq-C_{1} \mathcal{E}(t),
$$


onde $C_{1}=\min \left\{2 \delta, 2\left(\sigma_{0}-\delta\right)\right\}>0$.

Mostremos agora a equivalência entre $H(t)$ e $\mathcal{E}(t)$. Com efeito, temos

$$
|H(t)-\mathcal{E}(t)| \leq \delta \int_{0}^{L}|u|\left|u_{t}\right| d x+\frac{\delta}{2} \int_{0}^{L}|\sigma(x)||u|^{2} d x .
$$

Daí, utilizando a Desigualdade de Young, a Desigualdade de Poincaré e a hipótese (5), segue que

$$
|H(t)-\mathcal{E}(t)| \leq \frac{\delta}{2} \int_{0}^{L} u_{t}^{2} d x+\frac{\delta C_{0}}{2}\left(1+\sigma_{1}\right) \int_{0}^{L} u_{x}^{2} d x
$$

Tomando $C_{2}=\max \left\{1, C_{0}\left(1+\sigma_{1}\right)\right\}>0$, obtemos

$$
|H(t)-\mathcal{E}(t)| \leq \delta C_{2} \mathcal{E}(t)
$$

e, portanto,

$$
\left(1-\delta C_{2}\right) \mathcal{E}(t) \leq H(t) \leq\left(1+\delta C_{2}\right) \mathcal{E}(t)
$$

Escolhendo $\delta$ pequeno de modo que, além de ser $0<$ $\delta<\sigma_{0}$, satisfaça $1-\delta C_{2}>0$, conclui-se que

$$
K_{1} \mathcal{E}(t) \leq H(t) \leq K_{2} \mathcal{E}(t),
$$

onde $K_{1}=1-\delta C_{2}>0$ e $K_{2}=1+\delta C_{2}>0$.

De (20) e (21) chega-se à desigualdade diferencial

$$
\frac{d}{d t} H(t) \leq \frac{-C_{1}}{K_{2}} H(t),
$$

da qual se obtém

$$
H(t) \leq H(0) \exp \left(\frac{-C_{1}}{K_{2}} t\right)
$$

Combinando a desigualdade acima com a (21), concluímos que

$$
\mathcal{E}(t) \leq C \mathcal{E}(0) \exp (-\gamma t), \quad \forall t \geq 0,
$$

onde $C=\frac{K_{2}}{K_{1}}$ e $\gamma=\frac{C_{1}}{K_{2}}$, provando assim o teorema.

Nosso próximo passo, objetivo central deste trabalho, é o estudo do comportamento assintótico do par solução $(u, \theta)$ do sistema (1)-(4). Algumas considerações e resultados preliminares são necessários antes da apresentação do principal resultado.

Multiplicando ambos os membros da equação (1) por $\beta u_{t}$ e da equação (2) por $\alpha \theta$, adicionando ambas e integrando em $(0, L)$, obtemos

$$
\begin{aligned}
& \beta \int_{0}^{L} u_{t t} u_{t} d x-\beta \int_{0}^{L} u_{x x} u_{t} d x+\beta \int_{0}^{L} \sigma(x) u_{t}^{2} d x \\
& +\alpha \beta \int_{0}^{L} \theta_{x} u_{t} d x+\alpha \int_{0}^{L} \theta_{t} \theta d x-\alpha \int_{0}^{L} \theta_{x x} \theta d x
\end{aligned}
$$

$$
+\alpha \beta \int_{0}^{L} u_{x t} \theta d x=0
$$

Via integração por partes, chega-se facilmente à identidade

$$
\begin{array}{r}
\frac{1}{2} \frac{d}{d t} \int_{0}^{L}\left(u_{t}^{2}+u_{x}^{2}+\frac{\alpha}{\beta} \theta^{2}\right) d x= \\
\quad-\int_{0}^{L} \sigma(x) u_{t}^{2} d x-\frac{\alpha}{\beta} \int_{0}^{L} \theta_{x}^{2} d x .
\end{array}
$$

A expressão

$$
\mathcal{E}(t) \equiv \frac{1}{2} \int_{0}^{L}\left(u_{t}^{2}+u_{x}^{2}+\frac{\alpha}{\beta} \theta^{2}\right) d x
$$

é a Energia Total associada ao sistema. Tem-se, então,

$$
\frac{d}{d t} \mathcal{E}(t)=-\int_{0}^{L} \sigma(x) u_{t}^{2} d x-\frac{\alpha}{\beta} \int_{0}^{L} \theta_{x}^{2} d x, \forall t>0,
$$

o que nos leva a concluir que a energia é decrescente. Estamos interessados, pois, em analisar a forma que ocorre esse decaimento. O método utilizado é aquele empregado no início desta seção, quando se obteve o decaimento da energia da equação da onda com dissipação. Com o intuito de encontar a perturbação adequada para a Energia, vamos multiplicar ambos os membros da equação (1) por $u$ e integrar em $(0, L)$. Obtemos, com isso,

$$
\begin{aligned}
& \int_{0}^{L} u_{t t} u d x-\int_{0}^{L} u_{x x} u d x \\
& +\int_{0}^{L} \sigma(x) u_{t} u d x+\alpha \int_{0}^{L} \theta_{x} u d x=0 .
\end{aligned}
$$

Daí, integrando-se por partes o segundo e o quarto termos e utilizando-se as condições de contorno (4), chega-se a

$$
\begin{aligned}
& \frac{d}{d t}\left\{\int_{0}^{L} u_{t} u d x+\frac{1}{2} \int_{0}^{L} \sigma(x) u^{2} d x\right\}=-\int_{0}^{L} u_{x}^{2} d x \\
& \quad+\int_{0}^{L} u_{t}^{2} d x+\alpha \int_{0}^{L} \theta u_{x} d x .
\end{aligned}
$$

Consideremos, então, o funcional

$$
G(t)=\int_{0}^{L} u u_{t} d x+\frac{1}{2} \int_{0}^{L} \sigma(x) u^{2} d x
$$


Vale, com isso,

$$
\frac{d}{d t} G(t)=-\int_{0}^{L} u_{x}^{2} d x+\int_{0}^{L} u_{t}^{2} d x+\alpha \int_{0}^{L} \theta u_{x} d x .
$$

Definimos agora a Energia Perturbada $H(t)$ pondo

$$
H(t)=\mathcal{E}(t)+\delta G(t)
$$

onde $\delta$ é um parâmetro positivo a ser fixado.

Lema 3.2. Existe constante $C_{1}>0$ tal que

$$
\frac{d}{d t} H(t) \leq-C_{1} \mathcal{E}(t), \quad \forall t \geq 0 .
$$

Demonstração Levando-se em conta a Desigualdade de Poincaré e a hipótese (5) obtemos, de (23),

$$
\frac{d}{d t} \mathcal{E}(t) \leq-\sigma_{0} \int_{0}^{L} u_{t}^{2} d x-\frac{\alpha}{\beta C_{0}} \int_{0}^{L} \theta^{2} d x .
$$

Por outro lado temos, de (25), aplicando-se a Desigualdade de Young,

$$
\frac{d}{d t} G(t) \leq-\frac{1}{2} \int_{0}^{L} u_{x}^{2} d x+\int_{0}^{L} u_{t}^{2} d x+\frac{\alpha^{2}}{2} \int_{0}^{L} \theta^{2} d x .
$$

Segue, de (27)-(28), que

$$
\begin{aligned}
\frac{d}{d t} H(t) & \leq-2\left(\sigma_{0}-\delta\right) \frac{1}{2} \int_{0}^{L} u_{t}^{2} d x-\frac{\delta}{2} \int_{0}^{L} u_{x}^{2} d x \\
& -\left(\frac{2}{C_{0}}-\delta \alpha \beta\right) \frac{1}{2} \frac{\alpha}{\beta} \int_{0}^{L} \theta^{2} d x .
\end{aligned}
$$

Agora é só escolher $\delta>0$ suficientemente pequeno de modo que

$$
\sigma_{0}-\delta>0 \text { e } \frac{2}{C_{0}}-\alpha \beta \delta>0
$$

e fazer

$$
C_{1}=\min \left\{2\left(\sigma_{0}-\delta\right), \delta, \frac{2}{C_{0}}-\delta \alpha \beta\right\}
$$

que teremos

$$
\frac{d}{d t} H(t) \leq-C_{1} \mathcal{E}(t), \quad \forall t \geq 0,
$$

provando o desejado.

Lema 3.3. Existem constantes $K_{1}, K_{2}>0$ tais que

$$
K_{1} \mathcal{E}(t) \leq H(t) \leq K_{2} \mathcal{E}(t), \quad \forall t \geq 0 .
$$

Demonstração De (26), utilizando a hipótese (5), a Desigualdade de Hölder e a Desigualdade de Poincaré, segue que

$$
\begin{aligned}
|H(t)-\mathcal{E}(t)| & \leq \delta\left(\int_{0}^{L}|u|\left|u_{t}\right| d x+\frac{\sigma_{1}}{2} \int_{0}^{L}|u|^{2} d x\right) \\
& \leq \frac{\delta}{2} \int_{0}^{L} u_{t}^{2} d x+\left(1+\sigma_{1}\right) \frac{\delta}{2} \int_{0}^{L} u^{2} d x \\
& \leq \frac{\delta}{2} \int_{0}^{L} u_{t}^{2} d x+C_{0}\left(1+\sigma_{1}\right) \frac{\delta}{2} \int_{0}^{L} u_{x}^{2} d x
\end{aligned}
$$

e, portanto,

$$
\begin{aligned}
& (1-\delta) \frac{1}{2} \int_{0}^{L} u_{t}^{2} d x+\left[1-C_{0}\left(1+\sigma_{1}\right) \delta\right] \frac{1}{2} \int_{0}^{L} u_{x}^{2} d x \\
& +\frac{1}{2} \frac{\alpha}{\beta} \int_{0}^{L} \theta^{2} d x \leq H(t) \leq(1+\delta) \frac{1}{2} \int_{0}^{L} u_{t}^{2} d x \\
& +\left[1+C_{0}\left(1+\sigma_{1}\right) \delta\right] \frac{1}{2} \int_{0}^{L} u_{x}^{2} d x+\frac{1}{2} \frac{\alpha}{\beta} \int_{0}^{L} \theta^{2} d x .
\end{aligned}
$$

Tomando $\delta>0$ suficientemente pequeno de modo que, além de (29), satisfaça também

$$
1-C_{0}\left(1+\sigma_{1}\right) \delta>0 \text { e } 1-\delta>0,
$$

obtemos

$$
K_{1} \mathcal{E}(t) \leq H(t) \leq K_{2} \mathcal{E}(t)
$$

onde

$$
K_{1}=\min \left\{\left(1-C_{0}\left(1+\sigma_{1}\right) \delta\right), 1-\delta\right\}>0
$$

e

$$
K_{2}=\max \left\{\left(1+C_{0}\left(1+\sigma_{1}\right) \delta\right), 1+\delta\right\}>0 .
$$

Finalmente, o próximo resultado mostra que a energia total do sistema decai exponencialmente a zero quando $t \rightarrow \infty$.

Teorema 3.4. Seja $\mathcal{E}(t)$ a Energia do sistema (1)-(4), dada pela expressão (22). Então existem constantes $C, \gamma>0$ tais que

$$
\mathcal{E}(t) \leq C \mathcal{E}(0) \exp (-\gamma t), \forall t \geq 0 .
$$

Demonstração Dos Lemas 3.2 e 3.3 sabemos que

$$
\frac{d}{d t} H(t) \leq-C_{1} \mathcal{E}(t) \leq-\frac{C_{1}}{K_{2}} H(t)
$$

e, portanto,

$$
H(t) \leq H(0) \exp \left(-\frac{C_{1}}{K_{2}} t\right) .
$$


Daí, utilizando novamente o Lema 3.3, obtemos

$$
\mathcal{E}(t) \leq \frac{K_{2}}{K_{1}} \mathcal{E}(0) \exp \left(-\frac{C_{1}}{K_{2}} t\right),
$$

isto é,

$$
\mathcal{E}(t) \leq C \mathcal{E}(0) \exp (-\gamma t)
$$

onde, $C=\frac{K_{2}}{K_{1}}$ e $\gamma=\frac{C_{1}}{K_{2}}$.

\section{Conclusões}

A teoria de Semigrupos de Operadores Lineares Limitados mostrou-se uma ferramenta poderosa na demonstração de existência e unicidade de solução do problema (1)-(4). Na verdade, tal teoria pode ser aplicada a problemas muito mais gerais, incluindo os não-lineares. Quanto ao comportamento assintótico da solução, o método empregado pode ser utilizado em problemas de evolução mais gerais, com outras condições de contorno. O que se precisa, em geral, é encontar multiplicadores mais adequados a cada situação considerada. Claro está que, dependendo do tipo de dissipação considerada no modelo (dissipação localizada ou dissipação na fornteira, por exemplo), o método pode requerer cálculos mais sofisticados. Fica, no entanto, a perspectiva futura de abordar modelos não-lineares associados ao sistema (1)-(2) bem como considerar outros mecanismos de dissipação da energia.

\section{Agradecimentos}

O primeiro autor (D. Dalmolin) gostaria de agradecer à CAPES pelo suporte financeiro recebido enquanto cursava o mestrado em Matemática da Universidade Federal de Santa Maria. Ambos autores agradecem aos revisores pelas valiosas sugestões de alterações para a melhoria deste trabalho.

\section{Referências}

Achenbach, J. D., 1973. Wave Propagation in Elastic Solids.North-Holland Publishing Company, New York.

Adams, R. A., 1975. Sobolev Spaces. Academic Press.

Brezis, H., 2010. Functional Analysis, Sobolev Spaces and Partial Differential Equations. Springer.

Ciarlet, P. G., 1993. Mathematical Elasticity. Vol. 1. North-Holland, Amsterdam.
Dafermos, C. M., 1968. On the existence and the asymptotic stability of solutions to the equations of linear thermoelasticity. Arch. Rational Mech. Anal. 29, 241-271.

Ferreira, M. V., Menzala, G. P., 2006. Energy decay for solutions to semilinear systems of elastic waves in exterior domains. Electronic Journal of Differential Equations 2006, 1-13.

Ferreira, M. V., Menzala, G. P., 2007. Uniform stabilization of an electromagnetic-elasticity problem in exterior domains. Discrete and Continuous Dynamical Systems 18 (4), 719-746.

Nowacki, W., 1962. Thermoelasticity. Pergamon Press, Oxford.

Pazy, A., 1983. Semigroups of linear operators and applications to partial differential equations. SpringerVerlag, New York.

Pereira, D. C., Menzala, G. P., 1989. Exponential decay of solutions to a coupled system of equations of linear thermoelasticity. Comp. Appl. Math. 8, 193-204.

Rivera, J. E. M., 1992. Energy decay rates in linear thermoelasticity. Funkcialaj Ekvacioj 35, 19-30. 